\title{
Effects of valsartan combined with a-lipoic acid on renal function in patients with diabetic nephropathy: a systematic review and meta-analysis
}

Fangfang Sun ${ }^{1 *} \mathbb{D}$, Deqi Jiang ${ }^{2}$ and Juanjuan $\mathrm{Cai}^{3}$

\begin{abstract}
Background: Diabetic nephropathy (DN) is one of the most serious microvascular complications of diabetes, valsartan and a-lipoic acid alone or in combination has been used for the treatment of patients with DN. However, some results in these clinical reports were still controversial. The purpose of this study was to evaluate the efficacy of valsartan combined with a-lipoic acid on renal function in patients with DN.

Methods: We searched the electronic databases including PubMed, Sciencedirect, EMBASE, Cochrane library, Chinese national knowledge infrastructure (CNKI) and Wanfang databases, and the publication deadline was limited to January 2020. Randomized controlled trials (RCTs) evaluating the effects of valsartan combined with a-lipoic acid in DN patients were included. Pooled estimates were conducted using a fixed or random effect model. The outcomes included urinary albumin excretion rate (UAER), and the level of urinary albumin, $\beta_{2}$-microglobulin ( $\beta_{2^{-}}$ $\mathrm{MG}$ ), hypersensitive C-reactive protein (hs-CRP) and oxidative stress.

Results: 11 studies with 1294 participants were included in this study. The pooled analysis indicated that a-lipoic acid combined with valsartan could remarkably reduce UAER $(P<0.00001, \mathrm{SMD}=-1.95,95 \% \mathrm{Cl}=-2.55$ to $-1.20 ; P=$ $0.03, \mathrm{SMD}=-0.85,95 \% \mathrm{Cl}=-1.59$ to -0.1$)$ and the level of urinary albumin $(P=0.001, \mathrm{SMD}=-1.48,95 \% \mathrm{Cl}=-2.38$ to $-0.58 ; P=0.01, \mathrm{SMD}=-1.67,95 \% \mathrm{Cl}=-3.00$ to -0.33$), \beta_{2}-\mathrm{MG}(P<0.001, \mathrm{SMD}=-2.59,95 \% \mathrm{Cl}=-3.78$ to $-1.40 ; P=$ $0.03, \mathrm{SMD}=-0.48,95 \% \mathrm{Cl}=-0.93$ to -0.04$)$ when compared with valsartan or lipoic acid monotherapy in patients with DN. However, there was no significant difference in the level of hs-CRP among the three therapies $(P=0.06$, $\mathrm{SMD}=-2.80,95 \% \mathrm{Cl}=-5.67$ to $0.07 ; P=0.10, \mathrm{SMD}=-0.42,95 \% \mathrm{Cl}=-0.92$ to 0.08$)$. In addition, a-lipoic acid combined with valsartan markedly increased the level of SOD $(P=0.03, \mathrm{SMD}=1.24,95 \% \mathrm{Cl}=0.32$ to $1.03 ; P=0.0002, \mathrm{SMD}=$ $0.68,95 \% \mathrm{Cl}=0.32$ to 1.03$)$ and $\mathrm{T}-\mathrm{AOC}(P<0.00001, \mathrm{SMD}=0.89,95 \% \mathrm{Cl}=0.62$ to $1.16 ; P=0.02, \mathrm{SMD}=0.58,95 \% \mathrm{Cl}=$ 0.10 to 1.07$)$, and reduced the level of $\mathrm{MDA}(P=0.0002, \mathrm{SMD}=-1.99,95 \% \mathrm{Cl}=-3.02$ to $-0.96 ; P=0.0001, \mathrm{SMD}=-0.69$, $95 \% \mathrm{Cl}=-1.04$ to -0.34$)$.
\end{abstract}

\footnotetext{
* Correspondence: fangfs0907@126.com

'Department of pharmacy, Hangzhou Third People's Hospital, Hangzhou 310009, China

Full list of author information is available at the end of the article
}

C C The Author(s). 2021 Open Access This article is licensed under a Creative Commons Attribution 4.0 International License, which permits use, sharing, adaptation, distribution and reproduction in any medium or format, as long as you give appropriate credit to the original author(s) and the source, provide a link to the Creative Commons licence, and indicate if changes were made. The images or other third party material in this article are included in the article's Creative Commons licence, unless indicated otherwise in a credit line to the material. If material is not included in the article's Creative Commons licence and your intended use is not permitted by statutory regulation or exceeds the permitted use, you will need to obtain permission directly from the copyright holder. To view a copy of this licence, visit http://creativecommons.org/licenses/by/4.0/ The Creative Commons Public Domain Dedication waiver (http://creativecommons.org/publicdomain/zero/1.0/) applies to the data made available in this article, unless otherwise stated in a credit line to the data. 
Conclusions: $a-$-lipoic acid combined with valsartan could significantly reduce the level of urinary albumin and oxidative stress, increase antioxidant capacity and alleviate renal function damage in patients with DN, and this will provide a reference for the selection of treatment drugs for DN.

Keywords: Diabetic nephropathy, Valsartan, a-Lipoic acid, Renal function, meta-analysis

\section{Background}

Diabetic nephropathy accounts for $20-30 \%$ of patients with diabetes mellitus (DM), which is a frequent microvascular complication of DM and one of the important causes of chronic renal failure [1]. At present, about 15\% of DN patients would develop end-stage renal disease, and eventually lead to death due to renal failure [2]. Most patients with DN have an insidious onset, no obvious clinical symptoms in the initial stage, but the disease deteriorates when persistent albuminuria emerges. The pathophysiologic mechanisms of DN are multi-factorial. Previous studies found that abnormal hemodynamics, oxidative stress response, genetic factors, activation of protein kinase $\mathrm{C}$ and renin angiotensin II, and proinflammatory reactions were all related to the occurrence and progression of DN [3]. Hyperglycemia in DM could lead to a series of metabolic, hemodynamic and biochemical alterations in renal tissues, including the increasing formation of advanced glycation end products and reactive oxygen species (ROS), extracellular matrix accumulation, growth factors/cytokines secretion, alteration in glomerular filtration rate which causes proteinuria, and all these changes might lead to DN [4]. In addition, hyperglycemia could increase the production of renin angiotensin II, resulting in the profibrogenic and inflammation of renal tissue [5]. Moreover, the progression of DN could heighten the risk of morbidity and mortality of cardiovascular diseases.

At present, a variety of treatments, including lowering blood sugar, blood pressure and blood fat, antiinflammatory, anti-oxidation, traditional Chinese medicine treatment, combination of traditional Chinese and western medicine, were all used to mitigate renal function damages in patients with DN. However, the drugs prove effective against $\mathrm{DN}$ are still limited. Clinically, the commonly used therapeutic drugs included angiotensin converting enzyme inhibitors, angiotensin II receptor antagonists, HMG-CoA reductase inhibitors, vitamin $\mathrm{D}$ and its analogs, protein kinase $\mathrm{C}$ inhibitors, antioxidants, etc. These drugs used alone or in combination could reduce the level of proteinuria and improved kidney function. In addition, sodium-glucose cotransporter 2(SGLT2) inhibitors had also been used to reduce the levels of microalbuminuria and albuminuria due to its anti-proteinuric effects [6].

Valsartan is a kind of angiotensin II receptor blockers (ARBs), which has been widely used in the treatment of hypertension and showed renoprotective and cardioprotective effects [7]. Valsartan could antagonize the binding of angiotensin II to its receptor, dilate the glomerular artery blood vessels and lower glomerular pressure, reducing protein filtration and inhibiting the production of proteinuria [8]. In addition, valsartan could down-regulate the expression of endothelin and cytokines, inhibit the proliferation of glomerular cells, promote the degradation of extracellular matrix and suppress the collagen synthesis, then delay glomerular sclerosis, thus boosting renal function [9]. Results in one RCT indicated that valsartan could lower the incidence of microalbuminuria, and without increasing the incidence of renal dysfunction in patients with glucose tolerance [10]. In streptozotocin and high fat diet induced DN mice, valsartan could alleviate renal podocyte injure and inhibit the expression of profibrotic growth factors and proinflammatory cytokines, decrease the accumulation of lipids and the production of albuminuria, improve glomerulosclerosis [11]. Moreover, valsartan combined with other drugs (such as angiotensinase inhibitors, antioxidants, prostaglandin E1, etc.) could also significantly reduce proteinuria and alleviate kidney damage in diabetic patients [12].

Lipoic acid or $\alpha$-lipoic acid is a powerful antioxidant that can remove a variety of oxidative stress products including hydroxyl radicals, singlet oxygen, nitric oxide radicals, hydroperoxides, hydroperoxides, and regenerate other antioxidants (such as vitamins C, vitamins and glutathione) to maintain normal antioxidant capacity [13]. Previous study indicated that lipoic acid could up-regulate the expression of Nrf-2mediated antioxidant genes and peroxisome proliferator activated receptors-regulated genes, enhance the antioxidant defense system, and had been used for the treatment of diabetic complications [14]. In mitochondria, lipoic acid synthase could produce $\alpha$-lipoic acid that participates in regulating the process of glucose oxidation and ATP generation, while reduction of lipoic acid synthase would increase oxidative stress response and accelerate the progression of DN [15]. Further study found that lipoic acid could lower the concentration of proteinuria and mitigate oxidative stress and renal damage in diabetic rats [16]. One RCT reported that lipoic acid combined with pyridoxine could reduce oxidative stress and albuminuria in patients with DN [17]. 
In addition, the combination treatment of lipoic acid and ARBs (such as valsartan, telmisartan, losartan) has been used to bolster the renal function in patients with DN. However, the combined effects of these agents were still controversial. Moreover, few studies assess the effects of telmisartan or losartan combined with lipoic acid in the treatment of DN, while some clinical trials about the combination treatment of lipoic acid and valsartan have been reported. Therefore, we performed this metaanalysis to evaluate the efficacy of valsartan combined with lipoic acid on renal function in patients with DN.

\section{Methods}

\section{Literature search}

We conducted a systematic literature search according to the PRISMA guidelines, and the electronic databases included PubMed, Sciencedirect, Embase, Cochrane library, CNKI and Wanfang databases. All the databases were searched without language restrictions, and the studies were published up to January 2020. The keywords included valsartan or angiotensin II receptor blockers or ARBs, lipoic acid or $\alpha$-lipoic acid, DN or diabetes kidney disease, and randomized controlled trials.

\section{Study selection and exclusion criteria}

The included studies met the following criteria: a) patients were diagnosed with $\mathrm{DN}, \mathrm{b}$ ) the study designed to be a randomized controlled clinical trial, c) the studies evaluated the effects of valsartan combined with lipoic acid on renal functions, d) with treatment duration of at least 14 days, and full-text publications were available. The exclusion criteria are as follows: patients were diagnosed with DN and other complications; non-RCTs and protocol; studies with insufficient data.

\section{Data extraction}

All relevant data were separated using a customized data extraction table and performed by two independent reviewers. The information of extraction table included first authors' names and publication dates, types of studies, numbers of patients enrolled, the base characteristics of patients (including age and sex), interventions, duration of medication, dosage of valsartan and lipoic acid, and Jadad score. The clinical outcomes included UAER, urinary albumin, $\beta_{2}-\mathrm{MG}$, hs-CRP, SOD, malondialdehyde (MDA) and total antioxidant capacity(T-AOC).

\section{Quality assessment}

The Jadad scale was used to evaluate the quality of each selected study. The Jadad scores of the study ranged from 0 to 7 points, and the main contents included the methods of randomization, double blinding, allocation concealment, withdraw and dropouts. Any disagreements were resolved by discussion.

\section{Statistical analysis}

The data analysis was conducted using RevMan software 5.3, and random or fixed effect models were used to analyze the outcomes. The heterogeneity between studies was evaluated using the $I^{2}$ test. Significant heterogeneity was identified and random effect model was used when $I^{2} \geq 50 \%$ or $P$-value $\leq 0.05$. Otherwise the heterogeneity was low and fixed effect model was applied. For continuous outcomes, data were represented as standard mean difference (SMD) and 95\% confidence interval (CIs). Sensitivity analyses were conducted to exclude the mixed studies that might lead to potential bias. Publication bias was estimated using a funnel plot. All $P$-values were two-tailed, and significant statistical difference was considered when $P$ values $<0.05$.

\section{Results}

\section{Study description}

The process of study screening was shown in Figs. 1, 158 potentially relevant articles were sifted out through electronic databases. After removing duplicates, review, observational trials, meta-analysis and clinical guidelines, 18 articles were full-text assessed for eligibility. Then 7 of these researches were excluded because of non-RCT and unavailable data. Finally, 11 trials [18-28] met the included criteria and were selected in this study. The characteristics of all included studies were shown in Table 1. A total of 1249 patients with DN were enrolled, 549 patients received lipoic acid plus valsartan combination therapy, 554 patients were treated with valsartan, and the remaining 146 patients were treated with lipoic acid. The ages of all participants ranged from 44.4 to 71.93 years old. The daily doses of lipoic acid were 600 $\mathrm{mg}, 450 \mathrm{mg}$ or $300 \mathrm{mg}$, the dosage of valsartan was 80 $\mathrm{mg}$, and the treatment duration was 14 days. Among these trials, 11 trials compared the effects of valsartan with that of lipoic acid plus valsartan on renal function, 4 trials compared the effects of lipoic acid plus valsartan with that of lipoic acid on UAER. Five studies were of high quality, while six studies were of low quality.

\section{Clinical outcomes \\ Urinary albumin excretion rate (UAER)}

Ten studies evaluated the effects of lipoic acid plus valsartan and that of valsartan monotherapy on UAER (Fig. 2). Significant heterogeneity existed between the two groups, and random effect model was used $(P<$ 0.00001, $I^{2}=95 \%$ ). Compared with valsartan monotherapy, treatment with lipoic acid plus valsartan could significantly reduce the UAER in patients with DN $(P<$ $0.00001, \mathrm{SMD}=-1.95,95 \% \mathrm{CI}=-2.55$ to -1.20$)$. In addition, three studies compared the effects of lipoic acid plus valsartan with that of lipoic acid monotherapy on UAER. Random effect model was used because of 


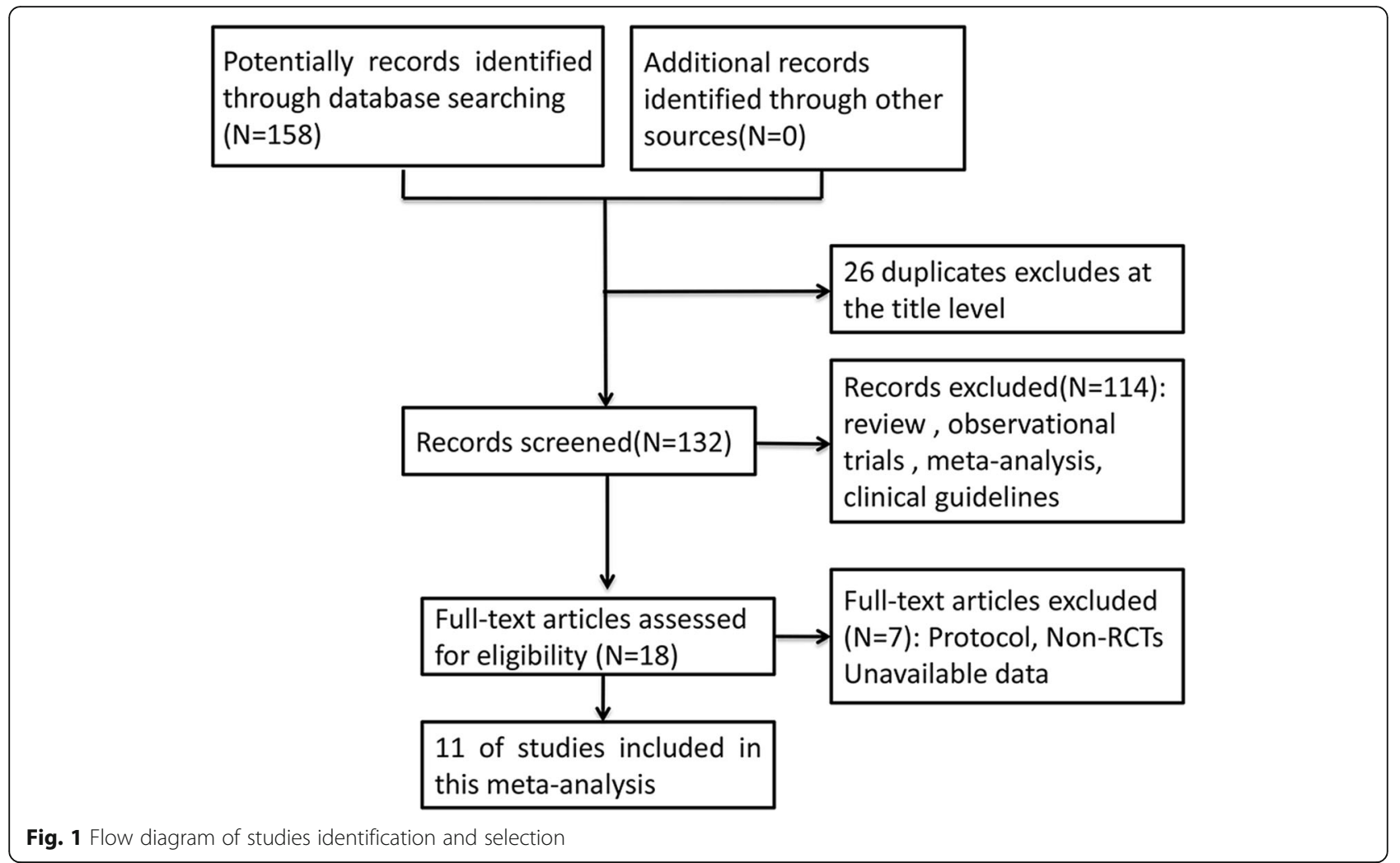

Table 1 Baseline characteristics of studies included in the meta-analysis

\begin{tabular}{|c|c|c|c|c|c|c|c|c|c|c|c|c|}
\hline \multirow{2}{*}{$\begin{array}{l}\text { Author and } \\
\text { year of } \\
\text { publication }\end{array}$} & \multirow[t]{2}{*}{$\begin{array}{l}\text { Study } \\
\text { design }\end{array}$} & \multicolumn{3}{|c|}{ sample size } & \multirow{2}{*}{$\begin{array}{l}\text { Age (mean } \pm \text { SD) } \\
\text { LV/V/L }\end{array}$} & \multirow{2}{*}{$\begin{array}{l}\text { Sex } \\
\text { male/ } \\
\text { female }\end{array}$} & \multirow[t]{2}{*}{$\begin{array}{l}\text { Duration } \\
\text { (days) }\end{array}$} & \multicolumn{2}{|c|}{$\begin{array}{l}\text { Dosage } \\
\text { (mg) }\end{array}$} & \multirow[b]{2}{*}{ Interventions } & \multirow[b]{2}{*}{ Outcomes } & \multirow[t]{2}{*}{$\begin{array}{l}\text { Jadad } \\
\text { scores }\end{array}$} \\
\hline & & LV & V & $\mathbf{L}$ & & & & $\mathbf{L}$ & V & & & \\
\hline $\begin{array}{l}\text { Chen M, } 201 \\
\text { 8[18] }\end{array}$ & $\mathrm{RCT}$ & 32 & 33 & 31 & $\begin{array}{l}71.41 \pm 7.52 / 70.92 \pm 7.24 / \\
71.93 \pm 7.05\end{array}$ & $48 / 48$ & 14 & 600 & 80 & LVN/L & (2)(4)(5)(6) & 3 \\
\hline $\begin{array}{l}\text { Deng GJ,201 } \\
\text { 8[19] }\end{array}$ & $\mathrm{RCT}$ & 40 & 40 & 40 & $\begin{array}{l}47.3 \pm 4.15 / 46.2 \pm 5.89 / 47.8 \pm \\
4.03\end{array}$ & $55 / 65$ & 14 & 300 & 80 & LVN/L & (1)(2) & 3 \\
\hline $\begin{array}{l}\text { Huo Y, } 201 \\
4[20]\end{array}$ & $\mathrm{RCT}$ & 60 & 60 & - & $44.4 \pm 4.2$ & $57 / 63$ & 14 & 600 & 80 & LVN & (1)(4)(5) & 4 \\
\hline $\begin{array}{l}\text { Jiang } Z Y, 201 \\
9[21]\end{array}$ & $\mathrm{RCT}$ & 51 & 51 & - & $69.21 \pm 3.54$ & $59 / 43$ & 14 & 600 & 80 & LVN & (1)(3)(4) & 4 \\
\hline Li Y, $2018[22]$ & $\mathrm{RCT}$ & 43 & 43 & - & $63.21 \pm 3.43 / 62.47 \pm 3.65$ & $52 / 34$ & 14 & 600 & 80 & LVN & (1)(2)(3) & 4 \\
\hline $\begin{array}{l}\operatorname{Lin} X J, 201 \\
3[23]\end{array}$ & RCT & 33 & 40 & 35 & - & $61 / 47$ & 14 & 600 & 80 & LVN/L & (1)(5)(6) & 2 \\
\hline Ma L, 201 9[24] & $\mathrm{RCT}$ & 48 & 48 & - & $57.26 \pm 4.31 / 57.84 \pm 3.92$ & $56 / 40$ & 14 & 600 & 80 & LVN & (1)(3) & 4 \\
\hline $\begin{array}{l}\text { Peng B, } 201 \\
3[25]\end{array}$ & $\mathrm{RCT}$ & 40 & 40 & 40 & - & - & 14 & 450 & 80 & LVN/L & (1) & 3 \\
\hline $\begin{array}{l}\text { Shi D, } 201 \\
8[26]\end{array}$ & $\mathrm{RCT}$ & 135 & 135 & - & $61.71 \pm 2.52 / 61.56 \pm 2.51$ & 131/139 & 14 & 600 & 80 & LVN & (1)(4)(5) & 3 \\
\hline $\begin{array}{l}\text { Wen WB,201 } \\
9[27]\end{array}$ & RCT & 26 & 25 & - & $56 \pm 11 / 56 \pm 9$ & $17 / 14$ & 14 & 300 & 80 & LVN & (1)(2) & 3 \\
\hline $\begin{array}{l}\text { Zhao J, } 201 \\
\text { 7[28] }\end{array}$ & $\mathrm{RCT}$ & 41 & 39 & - & $46.53 \pm 13.35 / 45.78 \pm 12.28$ & $50 / 30$ & 14 & 600 & 80 & LVN & (1)(3)(4)(5)(2) & 4 \\
\hline
\end{tabular}

Note: RCT, randomized controlled trial; L, lipoic acid; V, Valsartan; LV, lipoic acid+ Valsartan; (1)UAER = Urinary albumin excretion rate, (2)Urinary albumin, (3) $\beta 2-$ MG $=\beta 2$-microglobulin, (4)hs-CRP = Hypersensitive C-reactive protein, (5)SOD = Superoxide dismutase, (6MDA = malondialdehyde, (7)T-AOC = total antioxidant capacity 


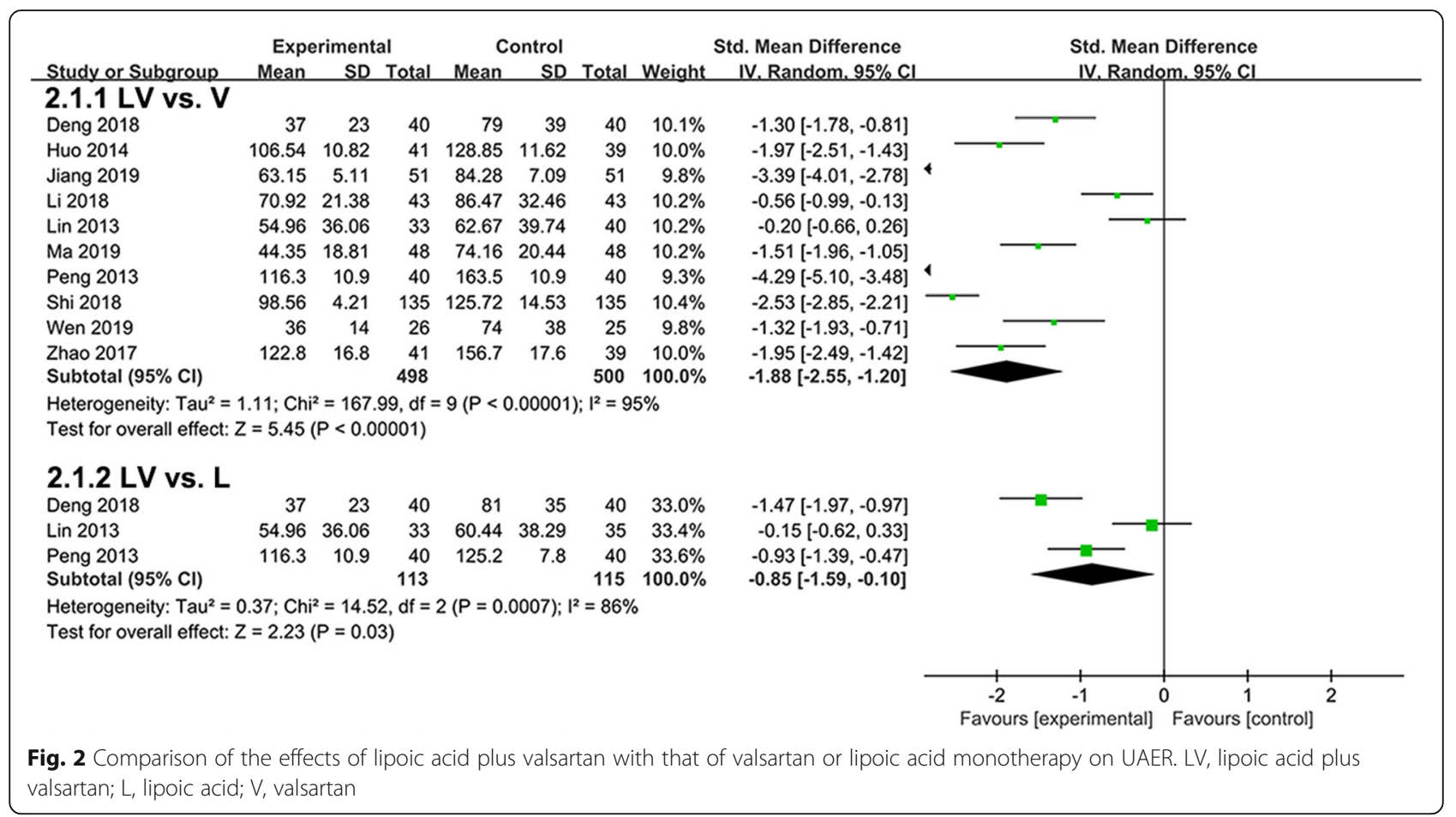

marked heterogeneity $\left(P=0.0007, I^{2}=86 \%\right)$. Treatment with lipoic acid plus valsartan was also superior to lipoic acid monotherapy in reducing UAER $(P=0.03$, SMD $=-$ $0.85,95 \% \mathrm{CI}=-1.59$ to -0.1 )

\section{Urinary albumin}

Four trials involving 353 patients compared the effects of lipoic acid combined with valsartan with that of monotherapy on the level of urinary albumin. The results were shown in Fig. 3, significant heterogeneity between the two groups was observed, and random effect models were used $\left(P<0.00001, I^{2}=91 \% ; P=0.0006\right.$, $\left.I^{2}=91 \%\right)$. Compared with valsartan or lipoic acid monotherapy, lipoic acid combined with valsartan could remarkably decrease the level of urinary albumin $(P=$ $0.001, \mathrm{SMD}=-1.48,95 \% \mathrm{CI}=-2.38$ to $-0.58 ; P=0.01$, $\mathrm{SMD}=-1.67,95 \% \mathrm{CI}=-3.00$ to -0.33$)$.

\section{$\beta_{2}$-microglobulin ( $\left.\beta_{2}-M G\right)$ and hypersensitive C-reactive protein (hs-CRP)}

Six studies evaluated the effects of lipoic acid combined with valsartan and that of monotherapy on the level of

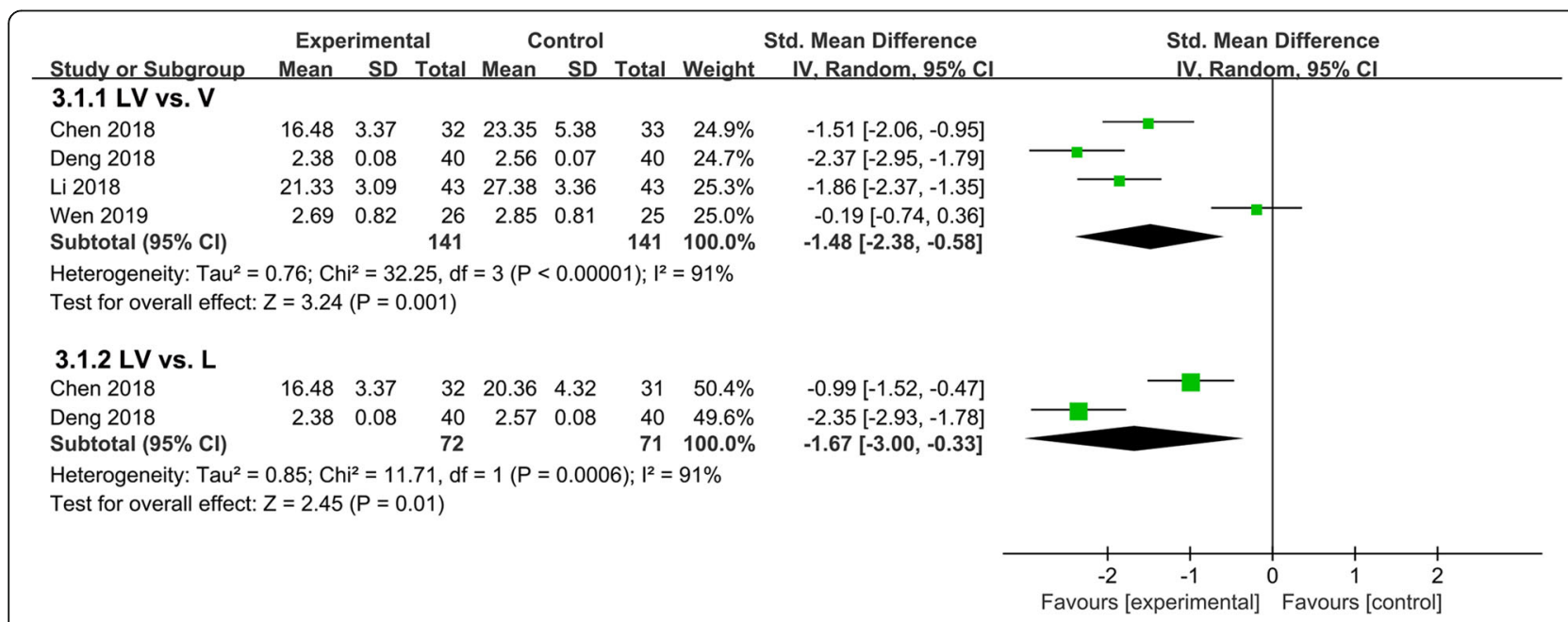

Fig. 3 Comparison of the effects of lipoic acid combined with valsartan with that of monotherapy on the level of urinary albumin. LV, lipoic acid plus valsartan; L, lipoic acid; $V$, valsartan 
$\beta_{2}-\mathrm{MG}$ in patients with DN. As shown in Fig. 4A, the pooled analysis indicated that heterogeneity was significant between the two groups $\left(P<0.00001, I^{2}=96 \%\right)$, and a remarkable decline in the level of $\beta_{2}$-MG in DN patients treated with lipoic acid plus valsartan was found $(P<0.001, \quad \mathrm{SMD}=-2.59, \quad 95 \% \mathrm{CI}=-3.78$ to $-1.40 ; \quad P=$ $0.03, \mathrm{SMD}=-0.48,95 \% \mathrm{CI}=-0.93$ to -0.04$)$.

Four studies involving 548 participants assessed the effects of lipoic acid combined with valsartan and that of monotherapy on the level of hs-CRP. We observed that there were no significant differences in hs-CRP level in DN patients receiving lipoic acid plus valsartan when compared with valsartan or lipoic acid monotherapy $(P=$ $0.06, \quad \mathrm{SMD}=-2.80,95 \% \mathrm{CI}=-5.67$ to $0.07 ; \quad P=0.10$, $\mathrm{SMD}=-0.42,95 \% \mathrm{CI}=-0.92$ to 0.08$)($ Fig. $4 \mathrm{~B})$.

\section{The levels of oxidative stress}

Oxidative stress was one of the important mechanisms of diabetic nephropathy, and the levels of SOD, MDA and T-
AOC in serum were all related to the process of renal impairments. Five trials evaluated the effects of lipoic acid combined with valsartan on the level of SOD in patients with DN (Fig. 5A). The results showed that lipoic acid combined with valsartan for the treatment of DN was much more effective in increasing the level of SOD than valsartan or lipoic acid monotherapy $(P=0.03, \mathrm{SMD}=$ $1.24,95 \% \mathrm{CI}=0.32$ to $1.03 ; P=0.0002, \quad \mathrm{SMD}=0.68$, $95 \% \mathrm{CI}=0.32$ to 1.03$)$. For MDA, the pooled results were showed in Fig. 5B. The effects of lipoic acid combined with valsartan treatments on the level of MDA were determined in four trials. Significant heterogeneity was observed among studies $\left(P<0.00001, I^{2}=92 \%\right)$. Remarkable reduction of MDA was witnessed in patients received lipoic acid plus valsartan $(P=0.0002, \quad S M D=-1.99$, $95 \% \mathrm{CI}=-3.02$ to $-0.96 ; P=0.0001, \mathrm{SMD}=-0.69,95 \% \mathrm{CI}=$ -1.04 to -0.34$)$. Three studies with 268 patients assessed the effects of lipoic acid and valsartan combination on TAOC. Statistical heterogeneity was not significant, and

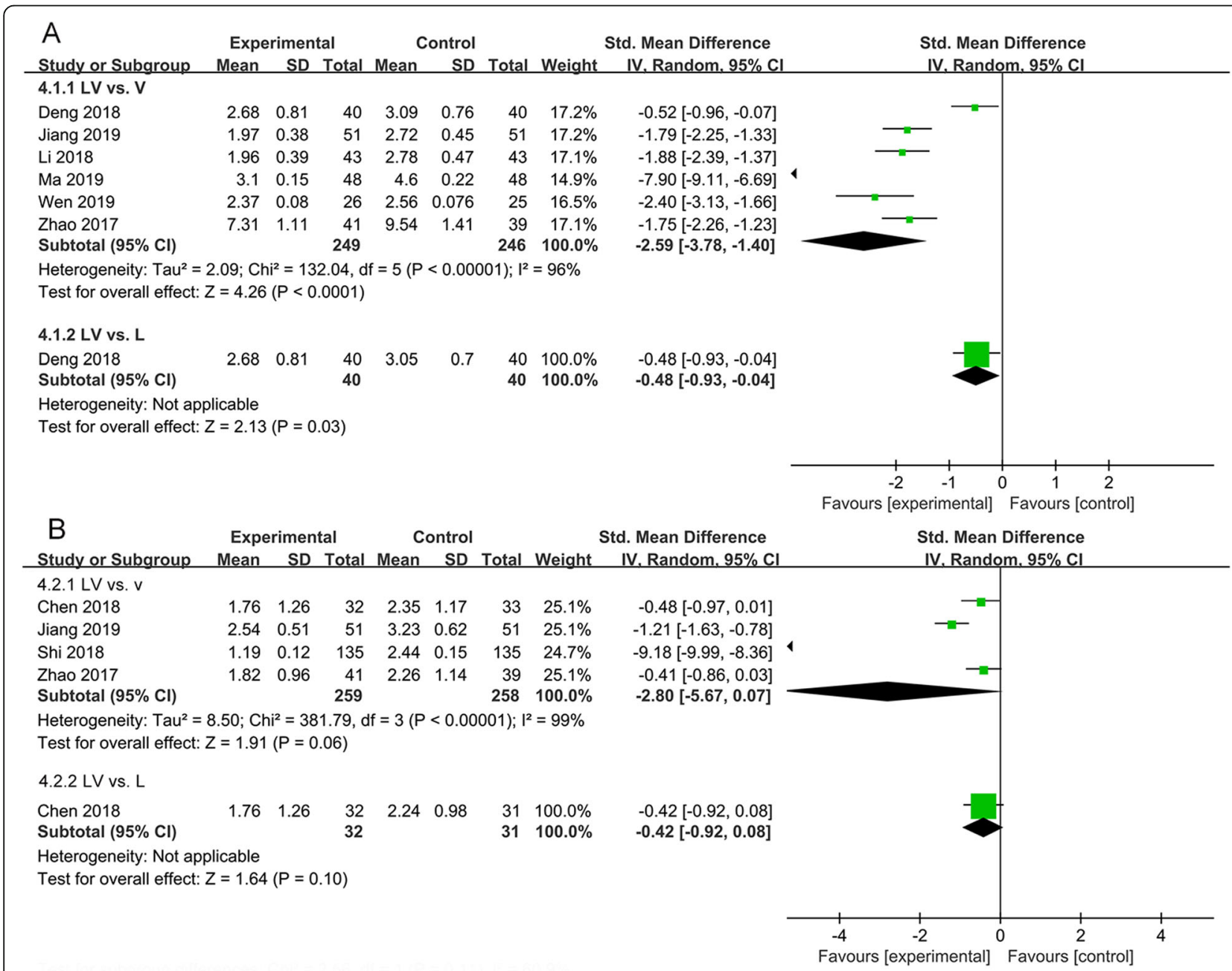

Fig. 4 Comparison of the effects of lipoic acid combined with valsartan with that of monotherapy on $\beta_{2}-M G$ and hs-CRP. (A) the level of $\beta_{2}-M G$; (B) the level of hs-CRP. LV, lipoic acid plus valsartan; L, lipoic acid; V, valsartan 


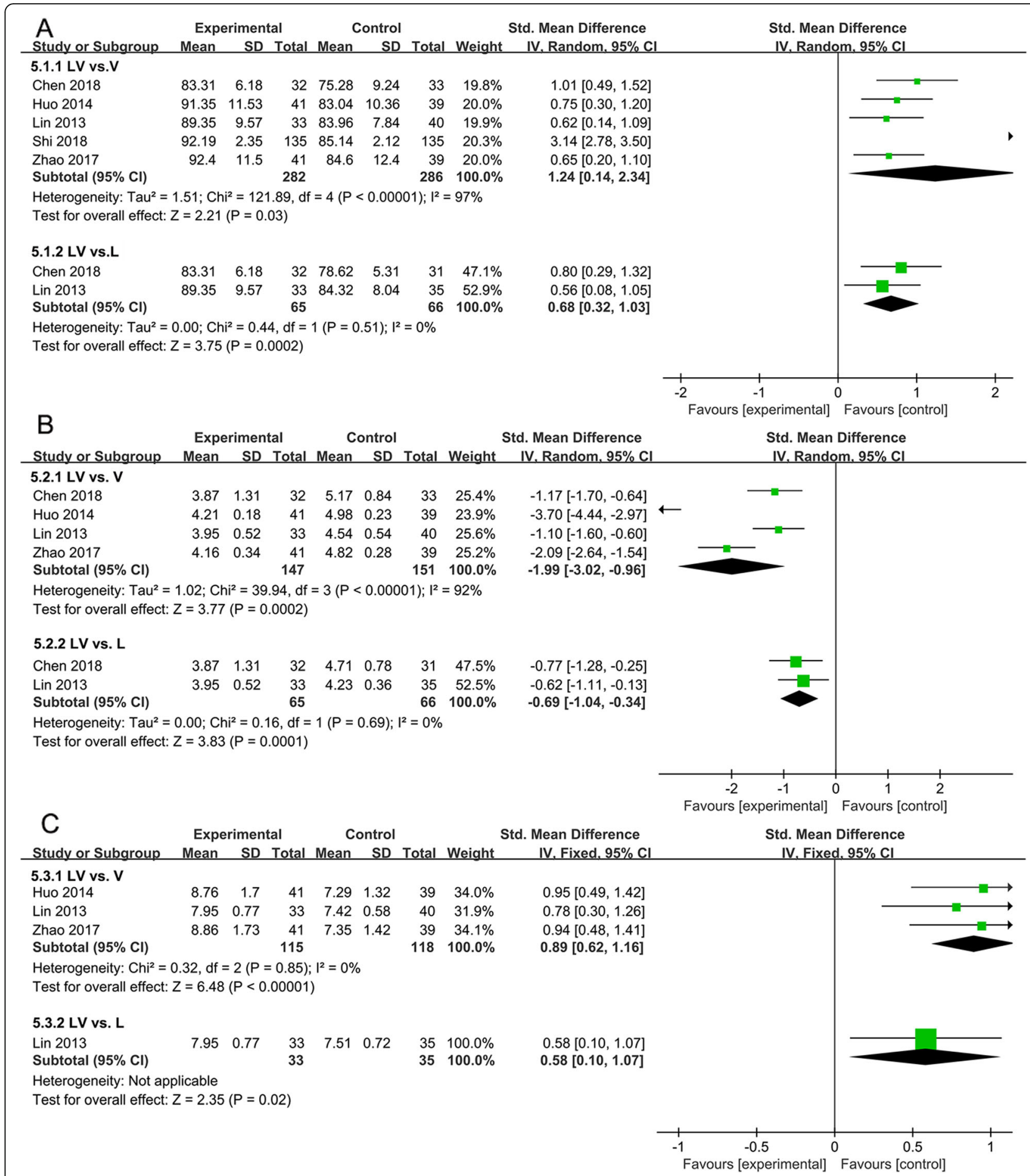

Fig. 5 Comparison of the effects of lipoic acid plus valsartan with that of valsartan or lipoic acid monotherapy on oxidative stress. (A) the level of SOD; (B) the level of MDA; (C) the levels of T-AOC. LV, lipoic acid plus valsartan; L, lipoic acid; V, valsartan

fixed effect model was applied $\left(P=0.85, I^{2}=0 \%\right)$ (Fig. 5C). Compared with valsartan or lipoic acid monotherapy, lipoic acid plus valsartan could markedly increase T-AOC levels $(P<0.00001, \mathrm{SMD}=0.89,95 \% \mathrm{CI}=0.62$ to $1.16 ; P=$ $0.02, \mathrm{SMD}=0.58,95 \% \mathrm{CI}=0.10$ to1.07).

\section{Sensitivity analysis and publication bias}

In the process of pooled analysis, we performed sensitivity analysis to evaluate heterogeneity. However, there was no significant difference in the value of $I^{2}$, and the values of SMD and the overall effects were close when 
changing the analysis model from fixed effect model to random effect model. The results of publication bias were showed in Fig. 6, the funnel plot was not symmetrical, indicating the existence of potential publication bias.

\section{Discussion}

$\mathrm{DN}$ is one of the major causes of end-stage renal failure, and the pathogenesis is related with the thickening of tubular and glomerular basement membrane, glomerulosclerosis and glomerular hypertrophy, endothelial dysfunction [29]. The main clinical feature of DN is the decline of glomerular filtration rate that leads to proteinuria [30]. At present, many clinical trials have been conducted to evaluate the effects of lipoic acid and valsartan used alone or in combination for the treatment of $\mathrm{DN}$, but some of the results remained controversial. Therefore, we performed this meta-analysis to provide solid evidence for the selection of drugs for the treatment of DN. Moreover, as far as we know, this is the first systematic analysis to comprehensively evaluate the treating effects of lipoic acid combined with valsartan on renal functions.

Microalbuminuria is considered as an important marker for the diagnosis and assessment of DN progression [31]. In patients with $\mathrm{DN}$, dysfunction of the glomeruli could boost urinary albumin excretion rate, cause excessive excretion of albumin in urine and bolster the production of microalbuminuria. However, renal function impairment also emerged among some diabetic patients in the absence of microalbuminuria [32]. Valsartan belongs to the class of angiotensin receptor antagonists, and is commonly used in the treatment of hypertension. It has certain protective effects on glomeruli by inhibiting angiotensin. Evidences from previous studies suggested that valsartan could remarkably lower the glomerular filtration rate and the production of proteinuria, playing a protective role in renal function in patients with type 2 diabetes [33,34]. $\alpha$-lipoic acid is an antioxidant that can protect $\beta$ cells, lower the level of cholesterol, reduce proteinuria and mesangial expansion in diabetic mice, thus inhibiting the development and progression of DN [35]. Additionally, $\alpha$-lipoic acid combined with alprostadil could decrease the levels of serum inflammatory factors and improve renal function of patients with DN [36]. In our study, the pooled analysis indicated that lipoic acid combined with valsartan was superior in lowering UAER, the level of urinary albumin and $\beta_{2}$-MG when compared with lipoic acid or valsartan monotherapy for the treatment of DN. The results were consistent with previous study [21].

Oxidative stress is a kind of stress response when the body is subjected to various harmful stimuli, and eventually causes damage to various cells, tissues and organs of the body. Current research found that oxidative stress was associated with the pathophysiology of DN [37]. Persistent hyperglycaemia caused the overproduction of reactive oxygen species (ROS) and activation of inflammatory mediators, inhibiting antioxidant defense mechanisms and eventually leading to oxidative stress which

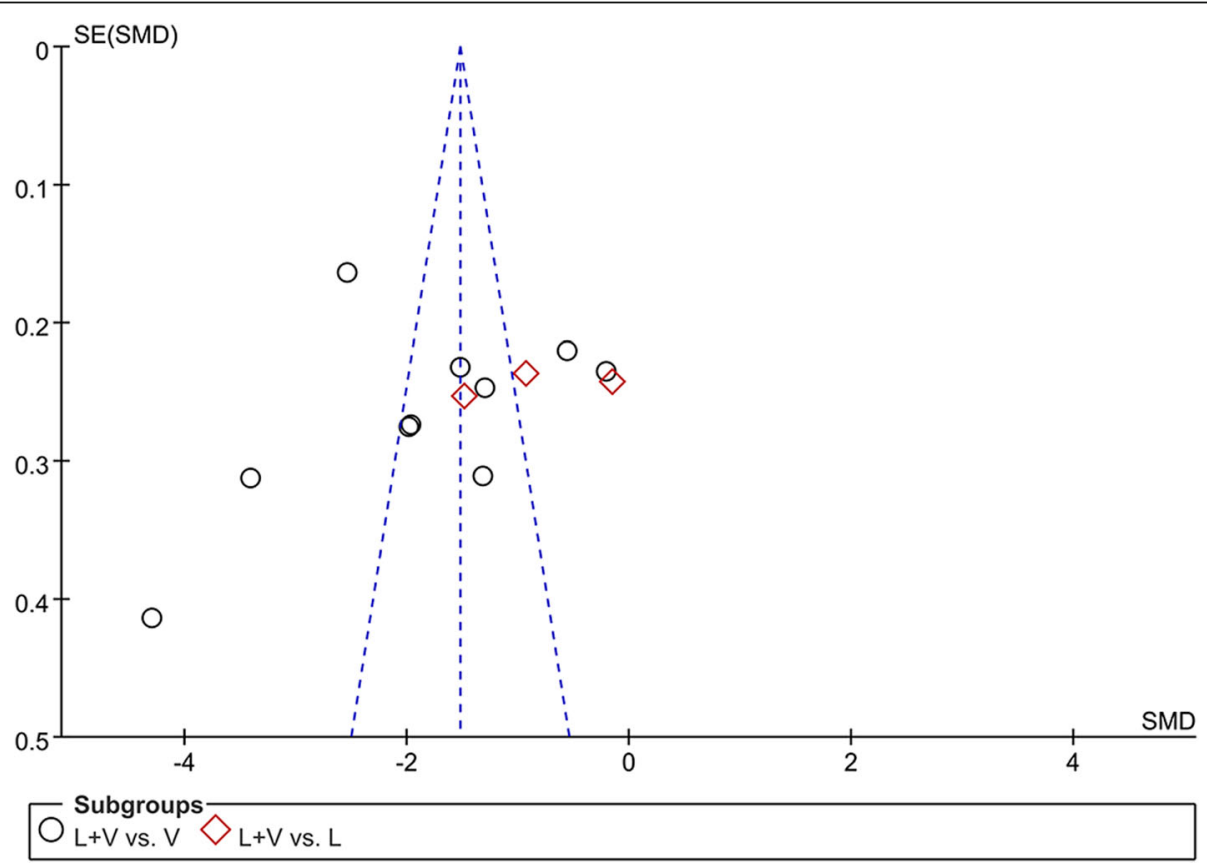

Fig. 6 Funnel plot for evaluating the risk of bias in the meta-analysis. LV, lipoic acid plus valsartan; L, lipoic acid; V, valsartan 
resulted in injury in the vessels and kidneys of diabetic patients [38]. Moreover, oxidative stress could lead to the abnormalities of hematological indices which might be used to predict the progression of DN. MDA and advanced oxidation products of protein (AOPP) as the peroxidation products of lipids and proteins could reflect the level of oxidative stress, and SOD and T-AOC are indicators that reflect the antioxidant capacity of the body [39]. Other study showed that valsartan could reduce the level of MDA and 8-hydroxy-deoxy guanosine, inhibit cyclosporine-A induced oxidative stress and alleviate the renal damages [40].

Lipoic acid acts as an antioxidant that can reduce oxidative stress, and it also has significant therapeutic effects on cardiovascular diseases, diabetes and its complications and neurodegenerative diseases. In alloxan-induced diabetic rabbits, low doses of lipoic acid significantly lowered the concentration of urine albumin, ameliorated oxidative stress and renal injury, so the agent proved to be effective in the treatment of diabetes and DN [16]. Later, another study further confirmed that the levels of urinary MDA and creatinine $(\mathrm{Cr})$ rose significantly, the ratios of SOD and serum glutathione peroxidase (SGSH-Px) were markedly decreased in patients with diabetes; $\alpha$-lipoic acid could significantly lower the ratios of MDA and $\mathrm{Cr}$, elevate the levels of SOD and SGSH-Px by inhibiting oxidative stress, and provide protection against glomerular podocyte injury [41]. In the present study, our results indicated that lipoic acid combined with valsartan could significantly increase the levels of SOD and T-AOC, decrease the level of MDA when compared with valsartan or lipoic acid monotherapy. From these results, we inferred that combination therapy of lipoic acid and valsartan inhibited oxidative stress, and enhanced antioxidant capacity.

Although the combination therapy proved to be more effective than each of the monotherapy, several possible limitations should still be taken into consideration to facilitate deeper research in the future. First, most of these included trials had relatively low sample sizes, which might be insufficient and our conclusions might not be flawless. Second, significant heterogeneity was observed between these studies, and sensitivity analysis was used to determine potential heterogeneity. Third, potential publication bias was existed, which might affect the accuracy of the results. Therefore, RCTs with large sample size and high quality were still needed to further confirm these conclusions.

\section{Conclusions}

Overall, eleven trials were included in this meta-analysis to assess the effects of lipoic acid combined with valsartan on renal functions in patients with $\mathrm{DN}$. The pooled findings indicated that lipoic acid combined with valsartan could significantly reduce the level of urinary albumin and oxidative stress, enhance antioxidant capacity and mitigate renal function damages in patients with $\mathrm{DN}$, and the findings proved to be helpful references for the selection of treatment drugs for $\mathrm{DN}$.

\section{Abbreviations \\ DN: Diabetic nephropathy; RCTs: Randomized controlled trials; DM: Diabetes mellitus; SGLT2: Sodium-glucose cotransporter 2; UAER: Urinary albumin excretion rate; $\beta_{2}-M G$ : $\beta_{2}$-microglobulin; hs-CRP: Hypersensitive C-reactive protein; MDA: Malondialdehyde; T-AOC: Total antioxidant capacity; \\ ROS: Reactive oxygen species}

\section{Acknowledgements}

Not applicable.

\section{Authors' contributions}

This article was put forward by FFS. DQJ and JJC designed this article and collected the data. FFS and DQJ performed the initial analysis and wrote the manuscript. All authors read and approved the final manuscript.

\section{Funding}

No

\section{Availability of data and materials}

All data generated or analyzed during this study are included in this published article, and you can find these data in references [18-28].

\section{Declarations}

Ethics approval and consent to participate

Not applicable.

Consent for publication

Not applicable.

\section{Competing interests}

The authors declare that they have no competing interests.

\section{Author details}

'Department of pharmacy, Hangzhou Third People's Hospital, Hangzhou 310009, China. ${ }^{2}$ Department of Biology and Pharmacy, Yulin Normal University, Yulin 537000, China. ${ }^{3}$ Department of Pathology, Zhejiang Province People's Hospital, Hangzhou 310014, China.

Received: 3 November 2020 Accepted: 19 August 2021

Published online: 31 August 2021

\section{References}

1. Vlachou E, Ntikoudi A, Govina O, Lavdaniti M, Kotsalas N, Tsartsalis A, et al. Effects of probiotics on diabetic nephropathy: a systematic review. Curr Clin Pharmacol. 2020;15(3):234-42. https://doi.org/10.2174/15748847156662003 03112753

2. Sagoo MK, Gnudi L. Diabetic nephropathy: an overview. Methods Mol Biol. 2020;2067:3-7. https://doi.org/10.1007/978-1-4939-9841-8_1.

3. Nagib AM, Elsayed Matter Y, Ashry Gheith O, Fathi Refaie A, Othman NF, AlOtaibi T. Diabetic nephropathy following posttransplant diabetes mellitus. Exp Clin Transplant. 2019;17(2):138-46. https://doi.org/10.6002/ect.2018.0157.

4. Wada J, Makino H. Innate immunity in diabetes and diabetic nephropathy. Nat Rev Nephrol. 2016;12(1):13-26. https://doi.org/10.1038/nrneph.2015.175.

5. Faselis C, Katsimardou A, Imprialos K, Deligkaris P, Kallistratos M, Dimitriadis K. Microvascular complications of type 2 diabetes mellitus. Curr Vasc Pharmacol. 2020;18(2):117-24. https://doi.org/10.2174/15701611176661 90502103733

6. Barutta F, Bernardi S, Gargiulo G, Durazzo M, Gruden G. SGLT2 inhibition to address the unmet needs in diabetic nephropathy. Diabetes-Metab Res. 2019;35:e3171.

7. Ecder T. Renal and metabolic effects of valsartan. Anadolu Kardiyol Derg. 2014;14(Suppl 2):S14-9. 
8. Suzuki K, Souda S, Ikarashi T, Kaneko S, Nakagawa O, Aizawa Y. Renoprotective effects of low-dose valsartan in type 2 diabetic patients with diabetic nephropathy. Diabetes Res Clin Pract. 2002;57(3):179-83. https://doi. org/10.1016/S0168-8227(02)00098-0.

9. Jacobsen P, Andersen S, Jensen BR, Parving HH. Additive effect of ACE inhibition and angiotensin II receptor blockade in type I diabetic patients with diabetic nephropathy. J Am Soc Nephrol. 2003;14(4):992-9. https://doi. org/10.1097/01.ASN.0000054495.96193.BF.

10. Currie G, Bethel MA, Holzhauer B, Haffner SM, Holman RR, McMurray J. Effect of valsartan on kidney outcomes in people with impaired glucose tolerance. Diabetes Obes Metab. 2017;19(6):791-9. https://doi.org/10.1111/ dom.12877.

11. Wang W, Qiu L, Howard A, Solis N, Li C, Wang X, et al. Protective effects of aliskiren and valsartan in mice with diabetic nephropathy. J Renin-AngioAldo S. 2014;15:384-95.

12. Black HR, Bailey J, Zappe D, Samuel R. Valsartan: more than a decade of experience. Drugs. 2009;69(17):2393-414. https://doi.org/10.2165/11319460000000000-00000.

13. Huk-Kolega H, Skibska B, Kleniewska P, Piechota A, Michalski L, Goraca A Role of lipoic acid in health and disease. Pol Merkur Lekarski. 2011;31(183): 183-5.

14. Golbidi S, Badran M, Laher I. Diabetes and alpha lipoic acid. Front Pharmacol. 2011:2:69.

15. Yi X, Xu L, Hiller S, Kim HS, Nickeleit V, James LR, et al. Reduced expression of lipoic acid synthase accelerates diabetic nephropathy. J Am Soc Nephrol. 2012;23(1):103-11. https://doi.org/10.1681/ASN.2011010003.

16. Winiarska K, Malinska D, Szymanski K, Dudziak M, Bryla J. Lipoic acid ameliorates oxidative stress and renal injury in alloxan diabetic rabbits. Biochimie. 2008;90(3):450-9. https://doi.org/10.1016/j.biochi.2007.11.010.

17. Noori N, Tabibi H, Hosseinpanah F, Hedayati M, Nafar M. Effects of combined lipoic acid and pyridoxine on albuminuria, advanced glycation end-products, and blood pressure in diabetic nephropathy. Int J Vitam Nutr Res. 2013;83(2):77-85. https://doi.org/10.1024/0300-9831/a000147.

18. Min C, Lixia C. Lipoic acid combined with valsartan for treatment of early diabetic nephropathy:impact on oxidative stress and inflammation. Chinese Journal of Clinicians. 2018:12:75-9.

19. Deng G. Clinical study on the treatment of type 2 diabetic nephropathy by combination of valsartan and alpha-sulfur acid. Journal of Anhui Health Vocational \& Technical College. 2016;15:53-4.

20. Yan H, Dezhi C, Zhaoling W. Lipoic acid combined with valsartan in the treatment of early diabetic nephropathy and its effect on oxidative stress. Chinese Journal of Primary Medicine and Pharmacy. 2014;21:489-91.

21. Jiang Z, Tan Z, Meng F, Li X. Curative effects of valsartan alone or combined with alpha-lipoic acid on inflammatory cytokines and renal function in early-stage diabetic kidney disease. J Coll Physicians Surg Pak. 2019;29(10): 1009-11. https://doi.org/10.29271/jcpsp.2019.10.1009

22. Yang $L$, Mingsong $G$, Caixia $K$, Fangxi $X$, Wei $L$, Zhenghua $Y$. Effect of valsartan combined with a-lipoic acid on early diabetic nephropathy and its effect on serum ET and $32-M G$ levels. Chinese Journal of Difficult and Complicated Cases. 2018;17:1217-20.

23. Xingjuan $\mathrm{L}$. The regulatory effect of a-lipoic acid and valsartan on oxidative stress in diabetic nephropathy. International Medicine and Health Guidance News. 2013;19:1161-5.

24. Li M, Quanguo Z, Yanyan L. Effect of a-lipoic acid combined with valsartan on vascular endothelial function and renal function in patients with diabetic nephropathy. International Medicine and Health Guidance News. 2019;17: 2862-4.

25. Peng B. Therapeutic effect of lipoic acid combined with valsartan on early diabetic nephropathy. Guide of China Medicine. 2011;11: 125-6.

26. Dai S, Long L, Bingbing Z, Xiaoxing W, Juan Y, Qian G, et al. Effect of lipoic acid combined with valsartan on early diabetic nephropathy. China Continuing Medical Education. 2018:10:120-1.

27. Weibin W, Fengying L. Clinical study of valsartan combined with a-lipoic acid in the treatment of early nephropathy of type 2 diabetes. Shanxi Medical Journal. 2010;39:534-5

28. Jing Z, Qijin W, Su W, Qingfeng L, Yan R. Analysis of efficacy and safety of lipoic acid and valsartan in the treatment of the early diabetic nephropathy. Progress in Modern Biomedicine. 2017;17:1931-3.

29. Kishore L, Kaur N, Singh R. Distinct biomarkers for early diagnosis of diabetic nephropathy. Curr Diabetes Rev. 2017:13(6):598-605. https://doi.org/10.21 74/1573399812666161207123007.
30. Flyvbjerg A. The role of the complement system in diabetic nephropathy. Nat Rev Nephrol. 2017;13(5):311-8. https://doi.org/10.1038/nrneph.2017.31.

31. Huang $M$, Liang $Q$, Li P, Xia J, Wang Y, Hu P, et al. Biomarkers for early diagnosis of type 2 diabetic nephropathy: a study based on an integrated biomarker system. Mol BioSyst. 2013;9(8):2134-41. https://doi.org/10.1039/c3 mb25543c.

32. Qi C, Mao X, Zhang Z, Wu H. Classification and differential diagnosis of diabetic nephropathy. J Diabetes Res. 2017;2017:8637138.

33. Ruggenenti $P$, Trillini M, P BD, Cortinovis M, Pisani A, Parvanova A, et al. Effects of valsartan, benazepril and their combination in overt nephropathy of type 2 diabetes: A prospective, randomized, controlled trial. Diabetes Obes Metab. 2019;21:1177-90.

34. Wang $Y$, Peng $W$, Zhang $X$, Qiao $H$, Wang $L$, Xu Z, et al. The association of ACE gene polymorphism with diabetic kidney disease and renoprotective efficacy of valsartan. J Renin-Angiotensin-Aldosterone Syst. 2016:17: 1470320316666749

35. Yi X, Nickeleit V, James LR, Maeda N. Alpha-lipoic acid protects diabetic apolipoprotein E-deficient mice from nephropathy. J Diabetes Complicat. 2011;25(3):193-201. https://doi.org/10.1016/j.jdiacomp.2010.07.004.

36. Hong Y, Peng J, Cai X, Zhang X, Liao Y, Lan L. Clinical efficacy of alprostadil combined with alpha-lipoic acid in the treatment of elderly patients with diabetic nephropathy. Open Med (Wars). 2017:12:323-7.

37. Sagoo MK, Gnudi L. Diabetic nephropathy: is there a role for oxidative stress? Free Radical Bio Med. 2018;116:50-63. https://doi.org/10.1016/j.freera dbiomed.2017.12.040.

38. Jha JC, Ho F, Dan C, Jandeleit-Dahm K. A causal link between oxidative stress and inflammation in cardiovascular and renal complications of diabetes. Clin Sci. 2018;132(16):1811-36. https://doi.org/10.1042/CS20171459.

39. Jha JC, Banal C, Chow BSM, Cooper ME, Jandeleit-Dahm K. Diabetes and kidney disease: role of oxidative stress. Antioxid Redox Sign. 2016;25(12): 657-84. https://doi.org/10.1089/ars.2016.6664.

40. Raeisi S, Ghorbanihaghjo A, Argani H, Dastmalchi S, Ghasemi B, Ghazizadeh $T$, et al. The effects of valsartan on renal klotho expression and oxidative stress in alleviation of cyclosporine nephrotoxicity. Transplantation. 2017; 101:e230.

41. Lin H, Ye S, Xu J, Wang W. The alpha-lipoic acid decreases urinary podocalyxin excretion in type 2 diabetics by inhibiting oxidative stress in vivo. J Diabetes Complicat. 2015;29(1):64-7. https://doi.org/10.1016/j.jdia comp.2014.09.011.

\section{Publisher's Note}

Springer Nature remains neutral with regard to jurisdictional claims in published maps and institutional affiliations.

Ready to submit your research? Choose BMC and benefit from:

- fast, convenient online submission

- thorough peer review by experienced researchers in your field

- rapid publication on acceptance

- support for research data, including large and complex data types

- gold Open Access which fosters wider collaboration and increased citations

- maximum visibility for your research: over $100 \mathrm{M}$ website views per year

At BMC, research is always in progress.

Learn more biomedcentral.com/submissions 